\title{
Temperature Distribution Reconstruction by Eigenfunction Interpolation of Boundary Measurement Data
}

\begin{abstract}
This paper treats the inverse problem of evaluating the temperature distribution over time in a composite solid material which unlike most of the other publications can have an arbitrary geometry. This approach is capable of evaluating the temperature over all the points within the domain of a nonhomogeneous object at every time instance. The method utilizes measurements in just few points of the peripheral surface of the geometry. The collected data are applied to estimate the weight coefficients of the numerically computed eigenfunctions of the problem which in turn leads to reconstruction of the temperature distribution everywhere.
\end{abstract}

Keywords-Eigenfunction; Inverse problem; Non-hmogenous; Temperature distribution; Complex geometry

\section{INTRODUCTION}

Monitoring and control heating processes require the possibility to infer in real time the temperature distribution, versus time and space, in the oven cavity region from the direct measurement of the local temperature measured in few spots located on the boundary or in proximity of the heating cavity. The temperature distribution reconstruction based on a small number of direct measurement point can be classified as an inverse problem, which is strongly underdetermined. Physical prior knowledge is thus necessary in order to be able to successfully invert the data and obtain the necessary temperature distribution.

Since the geometry and the material properties of the oven's components are well known and the physics of the heating process can be modeled by a proper set of partial differential equations (PDE) we can use this prior information for defining a set of basis function for the function space where the sought temperature distribution is defined.

In general the solution of the real physical problem cannot be attained analytically because of the complex geometry of real ovens and the composite, multilayered, anisotropic materials used for this kind of process.

In this paper we are proposing to interpolate the temperature spatial distribution using the physical problem eigenfunctions as interpolator base. The eigenfuntions, and the associated eigenvalues, are evaluated numerically using a commercial 3D multiphysics finite element method (FEM) code, customized for such type of computation.

Initially a simple problem, with known analytical solution, has been talked in order to be able to verify the numerical solver ability to compute the correct set of eigenfunctions. These results are presented in this extended abstract while the analysis of a real geometry will be introduced in the conference paper.

In literature the problem of heat conduction in an object made of materials with different densities, heat capacities and thermal conductivities is accounted as a very appealing subject. Unfortunately the majority of the presented solutions consider one dimension problems and the minority have described two or three-dimensional cases [1], [2]. However, the exact analytic solutions can only be derived for simple geometries as Salt [3] which has considered Cartesian system or Abdul Azeez and Vakakis [4] using cylindrical coordinates.

In [1] a two layer cylinder has been considered and the analytical solution for transient heat is presented taking advantage of symmetry of the problem the dimension is reduced to 2D axi-symmetric problem. The method of separation of variables is used in order to derive the solution but, still there are lots of difficulties finding the complex independent eigenvalues of the separated ordinary differential equation (ODE). Therefore, in this paper for analyzing the temperature distribution over a complex geometry -in general 3D- a FEM model has been implemented in order to numerically compute the eigenvalues and corresponding eigenfunctions. The temperature value in every point during time can be then reconstructed based on the evaluated eigenfunctions and related time dependent weight coefficients. In order to verify the results of the methodology the geometry described in [1] is selected and simulated, the results presented in the result section are consistent with the derived analytical solution. Since in the real situation the temperature data are acquired by sensors and the model parameters are not perfectly known, the robustness of the method has been tested and verified in presence of corrupting signals and parameters with stochastic noise.
Gabriele D'Antona

Department of Energy, Politecnico di Milano, 20133 Milan, Italy gabriele.dantona@polimi.it

Nima Seifnaraghi

Department of Energy, Politecnico di Milano, 20133 Milan, Italy nima.seifnaraghi@gmail.com 
In the following the methodology for setting the inverse problem and identifying the interpolating functions is introduced in section II; the first numerical result and performance analysis for a simple test case are presented in section III. The conclusion and a summary of activities that will be performed for the final conference paper are provided in section IV.

\section{METHODOLOGY}

The problem of heat equation can be considered as special case of general Strum-Liouville problems. Equation (1) shows a Strum-Liouville initial value boundary problem (IVBP) which for the sake of simplicity is written as one dimensional equation in the following, however, $x$ can be expanded to a vector of $m$ dimensions [5]:

$$
\begin{array}{rlrl}
\omega(x) \frac{\partial \mathrm{u}}{\partial t}-\frac{\partial}{\partial x}\left(P(x) \frac{\partial \mathrm{u}}{\partial x}\right)+R(x) u & =F(x, t) & a<x<b, t>0 \\
\alpha_{1} u(x)+\alpha_{2} \frac{d u}{d x}(x) & =0 & x & =a \\
\beta_{1} u(x)+\beta_{2} \frac{d u}{d x}(x) & =0 & x & =b
\end{array}
$$

Now if $\omega(x)$ is replaced with $\rho(x) c(x)$ and $P(x)=k(x)$ and $R(x)=0$, the equation will turn to heat equation with varying density $(\rho)$, specific heat $(c)$ and thermal conductivity $(k)$ with general boundary condition which by choosing $\alpha$ and $\beta$ can be used to describe either Dirichlet or Neumann boundary conditions. If the spatial operator $\mathrm{L}$ is defined as:

$$
L=\frac{1}{\rho(x) c(x)}\left(-\frac{d}{d x}\left(k(x) \frac{d}{d x}\right)\right)
$$

Then the eigenvalue problem will be:

$$
L \varphi=\lambda \varphi
$$

It's been proven in the literature [6] that the preceding eigenvalue problem (3) has finitely many real eigenvalues $\left(\lambda_{1, \ldots, n}\right)$ and corresponding orthogonal eigenfunctions $\varphi_{1, \ldots, n}$. Hence any square integrable function in m-dimensional space can be expressed as weighted summation of these $n$ independent orthogonal bases such as:

$$
T\left(x_{1}, \ldots, x_{m}, t\right)=\sum_{k=1}^{n} \alpha_{k}(t) \varphi_{k}\left(x_{1}, \ldots, x_{m}\right)
$$

where T represents the temperature distribution over the $\mathrm{m}$ dimensional space and $\alpha_{k}(t)$ are time dependent weight coefficients which are unknown. It is not possible to evaluate all the eigenvalues however, the first few smallest eigenvalues and related eigenfunctions will be enough to approximate the temperature quite precisely [5]. The last but not the least would be implementing a method in order to estimate the weight coefficients $\alpha_{k}(t)$. Numerically this can be done using an inversion method by measuring the temperature at few available points on the surface of the structure followed by solving an inverse problem which will be explained in more details during the given solution to a simple case study. The general steps of the solution for a two dimensional geometry would be as the following:

$$
T\left(x_{1}, x_{2}, t\right)=\sum_{v=1}^{n} \alpha_{k}(t) \varphi_{k}\left(x_{1}, x_{2}\right)
$$

If the temperature is measured at $j$ points:

$$
m_{j}(t)=T\left(x_{1, j}, x_{2, j}, t\right)=\sum_{k=1}^{n} \alpha_{k}(t) \varphi_{k}\left(x_{1, j}, x_{2, j}\right)
$$

where $x_{1, j}, x_{2, j}$ represent the spatial position of where the $j$ th sensor is located. The preceding equations can be written in the matrix form. Hence at a specific time $t=\bar{t}$ we have:

$$
\mathbf{m}=\mathbf{A} \alpha=\left[\begin{array}{ccc}
\varphi_{1}\left(x_{1,1}, x_{2,1}\right) & \ldots & \varphi_{n}\left(x_{1,1}, x_{2,1}\right) \\
\vdots & \vdots & \vdots \\
\varphi_{1}\left(x_{1, j}, x_{2, j}\right) & \ldots & \varphi_{n}\left(x_{1, j}, x_{2, j}\right)
\end{array}\right]\left[\begin{array}{c}
\alpha_{1}(\bar{t}) \\
\vdots \\
\alpha_{j}(\bar{t})
\end{array}\right]
$$

with

$$
\mathbf{m}=\left[\begin{array}{c}
m_{1}(\bar{t}) \\
\vdots \\
m_{j}(\bar{t})
\end{array}\right]
$$

Equation (7) can be inverted using the minimum norm solution in order to estimate the values of $\alpha_{k}$ :

$$
\overline{\boldsymbol{\alpha}}=\mathbf{A}^{T}\left(\mathbf{A} \mathbf{A}^{T}\right)^{-1} \mathbf{m}=\left[\begin{array}{c}
\overline{\alpha_{1}}(\bar{t}) \\
\vdots \\
\overline{\alpha_{n}}(\bar{t})
\end{array}\right]
$$

Once the coefficients are estimated the temperature distribution can be reconstructed as:

$$
\bar{T}\left(x_{1}, x_{2}, \bar{t}\right)=\sum_{\substack{k=1 \\ \text { III. }}}^{n} \overline{\alpha_{k}}(\bar{t}) \varphi_{k}\left(x_{1}, x_{2}\right)
$$

As a first test case was considered the simple physical system shown in Fig. 1 composed by two layer cylinder [1].A very short heating pulse carrying energy of $Q=100 \mathrm{~W} / \mathrm{m}^{2}$ with a radius of $d_{p}$ is radiated to the lower layer of the object. The boundary conditions at the top and bottom surfaces are considered as Neumann boundary conditions with the heat transfer coefficient of $h_{1,2}=50 \mathrm{~W} / \mathrm{m}^{2}$. K also for boundaries at $r=\mp R_{0}, h$ is assumed to be zero. The object is supposed to have an initial condition of zero $\mathrm{K}$ and constructed from a Titanium layer with $\rho_{1}=4940 \mathrm{Kg} / \mathrm{m}^{3}, c_{1}=710 \mathrm{~J} / \mathrm{Kg}$. K,$k_{1}=$ $7.5 \mathrm{~W} / \mathrm{m} . \mathrm{K}$ while $\rho_{2}=8700 \mathrm{Kg} / \mathrm{m}^{3}, c_{2}=385 \mathrm{~J} / \mathrm{Kg}$. K and $k_{2}=400$ $\mathrm{W} / \mathrm{m} . \mathrm{K} \cdot$

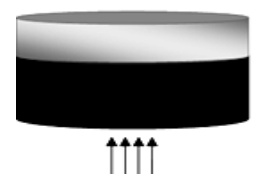

(a)

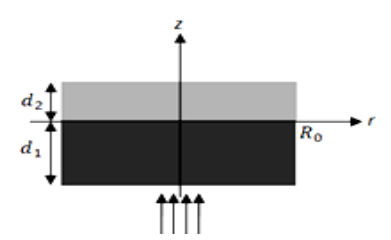

(b)
Figure 1. (a) Sample geometry in 3D; (b) 2D cross section of the sample

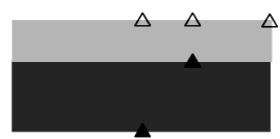

Figure 2. The hollow triangles stand for the measured points and the solid ones are the points where temperature is reconstructed 
are considered for the copper layer located over it. The pulse is radiated with a radius of $d_{p}=5 \mathrm{~mm}$ and the radius of the sample is $R_{0}=25 \mathrm{~mm}$. In addition for the sake of demonstration the thickness of the materials are assumed to be $d_{1}=d_{2}=0.5 \mathrm{~mm}$.

The first six smallest eigenvalues are selected and three points on the top surface are measured which can seen in Fig. 2. The heat transfer simulations and eigenfunction evaluations are done using Comsol Multiphysics and Matlab is employed for the remaining mathematical computations.

The first six eigenfuctions are demonstrated in Fig. 3 with their corresponding eigenvalues. Due to the fact that geometry is symmetric with respect to $\mathrm{z}$ axis the minus values of $r$ are not present in the plots.

After few tens of seconds the temperature of the system will be significantly low, however, the temperature distributed in upper layer will be more due to the lower heat capacity of the copper. One should notice as mentioned before the eigenfunctions corresponding to higher eigenvalues will decay in time hence as it is shown in Fig. 3.a the shape of eigenfunction related to smallest eigenvalue namely $\lambda=$ 0.0291 almost fully describe the long term behavior of the system.

The results of reconstructed temperature distribution along with the real temperature distribution are shown in Fig. 4. The two points are chosen to be different from the points where the weight coefficients were evaluated from.

The first point is considered on the surface with $(r, z)=(0$.$0.5) \mathrm{mm}$ and the second point is taken to be inside the geometry located at $(r, z)=(10,0) \mathrm{mm}$. In addition the temperature values in the measured spots are intentionally corrupted with a strong noise of $10 \%$ in order to take in to account the real case difficulties in utilizing sensors and to monitor whether the algorithm is robust or not.

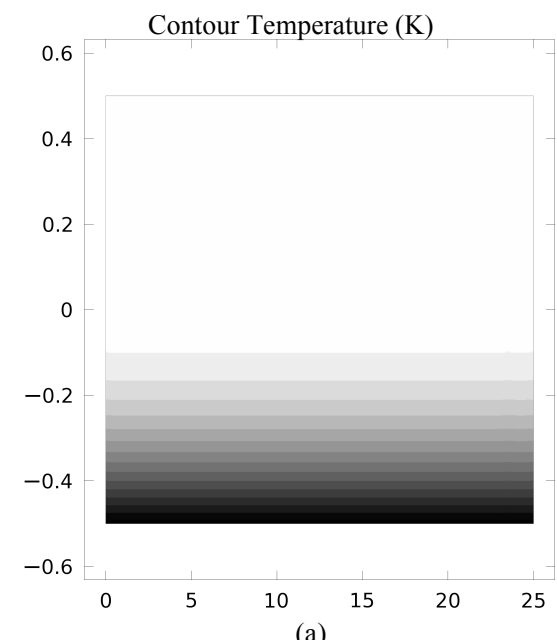

(a)

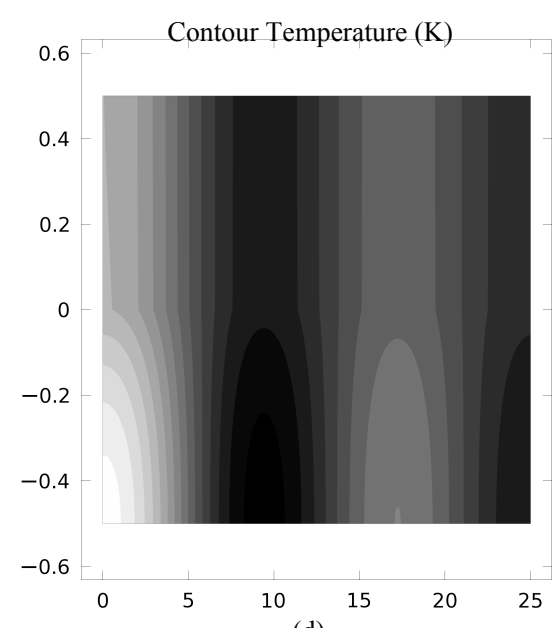

(d)

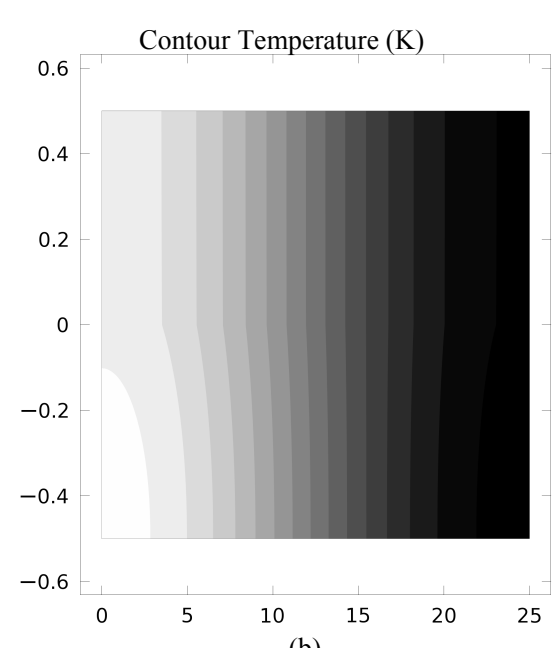

(b)

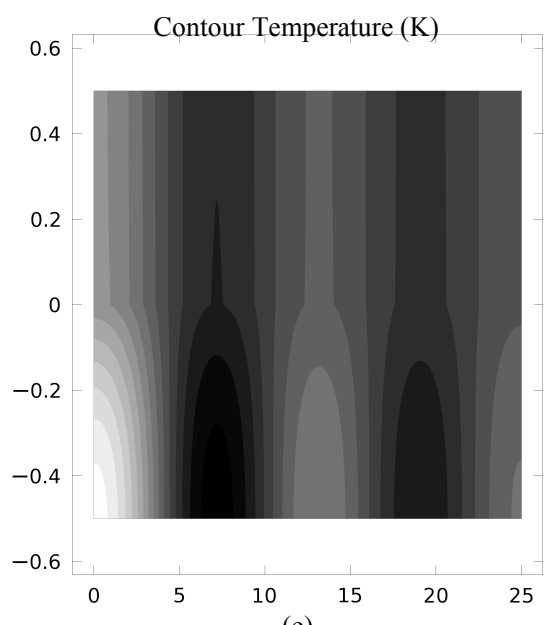

(e)

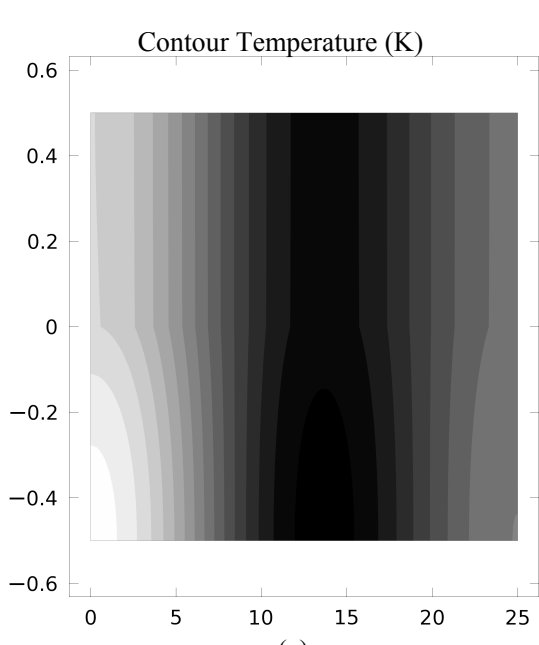

(c)

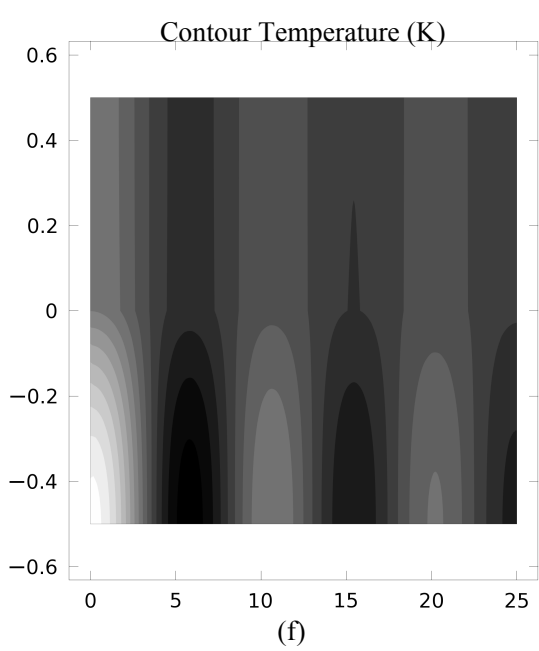

Figure 3. The shape of the first six eigenfuctions over the sample's domain. (a) $\lambda_{1}=0.0291 ;$ (b) $\lambda_{2}=1.388$; (c) $\lambda_{3}=4.290 ;\left(\right.$ d) $\lambda_{4}=8.02 ;$ (e) $\lambda_{5}=11.692$; (f) $\lambda_{6}=14.711$ 

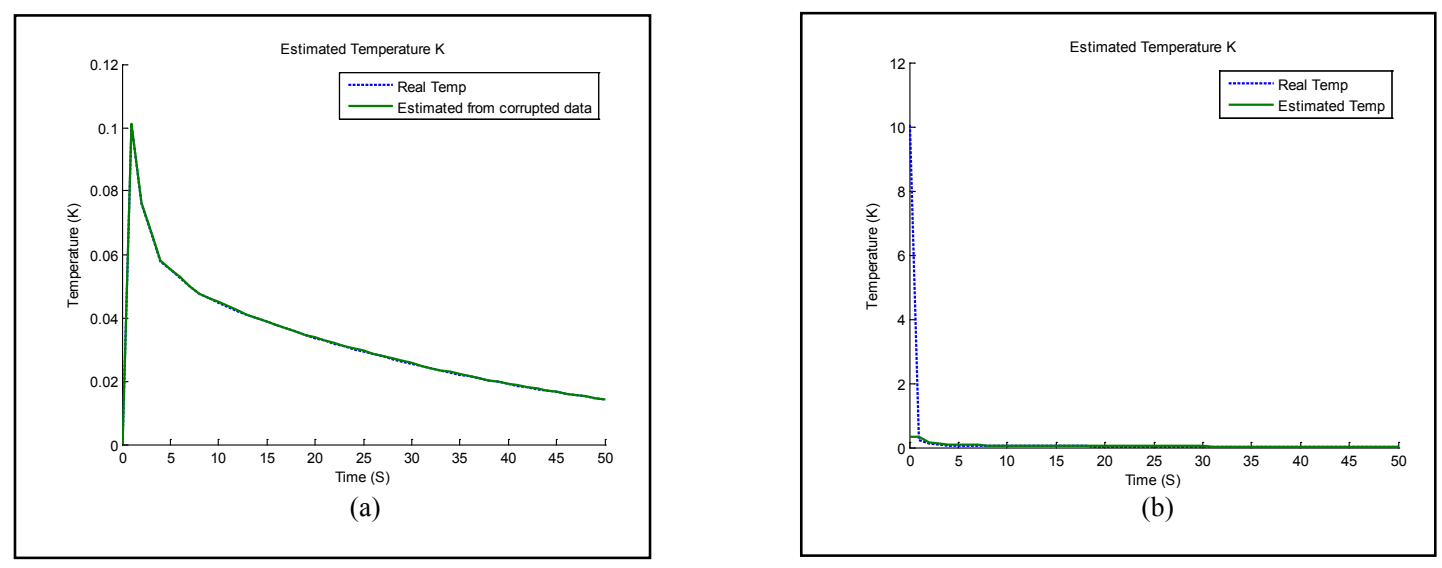

Figure 4. Real and estimated temperature neglecting the data corruption. (a) at point $(10,0)$; (b) at point $(0,0)$
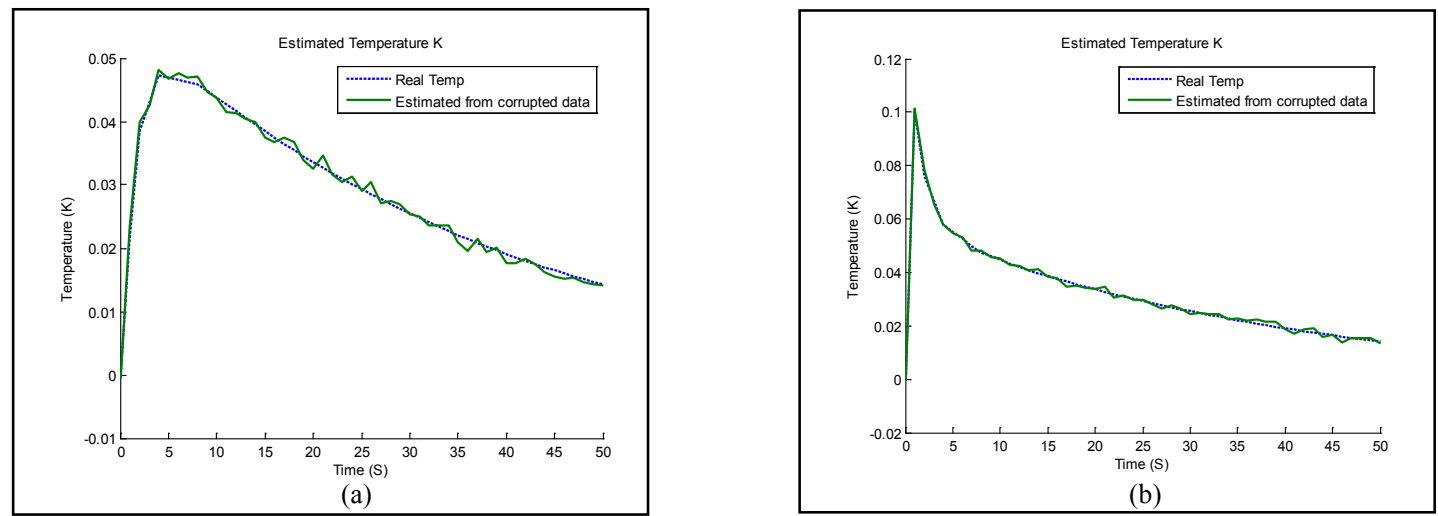

Figure 5. Real and estimated temperature considering the data corruption. (a) at point $(25,0.5)$; (b) at point $(10,0)$

The plots in Fig. 4 depict the temperature values over an interval of $50 \mathrm{~s}$. It should be noticed that since the selected point in Fig. 4.b is exactly located at the center of the radiated instantaneous pulse and the time steps that is considered for reconstructing the weight coefficients are one second which is quite large compared to interval of the pulse which is about fraction of half a second. Although, the consistency with the real temperature is quite clear from the first second onward. In Fig. 5 the temperature is estimated using the corrupted data in two points. The first one Fig. 5.a corresponds to one of the points that was utilized to compute the weight coefficients while the Fig. 5.b renders the temperature at the same point as in Fig. 4.a.

As a second example an induction cook top having a more complex geometry has been studied. It is believed that to have an efficient cooking procedure the temperature should be maintained at a certain level. Hence, in general producers are interested to have a quite accurate estimation of the temperature during time at the top surface of the work piece -where the to be cooked material will be placed eventually- in order to be able to design control circuit boards.

In this case the problem is solved in $3 \mathrm{D}$ and the axisymmetric feature of the geometry is ignored intentionally. The steel work piece having a thickness of $h_{w}=2 \mathrm{~mm}$ and a radius of $r_{w}=85 \mathrm{~mm}$ is placed on a glass surface with $h_{g}=5 \mathrm{~mm}$ and $r_{g}=170 \mathrm{~mm}$. The copper heat-coil consists of 15 turns, each carrying a current of $30 \mathrm{~A}$, alternating at $15 \mathrm{kHz}$. Outer radius and inner radius of the coil are $r_{o c}=85 \mathrm{~mm} r_{i c}=10 \mathrm{~mm}$ respectively. Ferrite disc has it's outer radius $r_{o f}=85 \mathrm{~mm}$ and inner of $r_{i f}=5 \mathrm{~mm}$.

Fig. 6 shows the cross section of the considered geometry. The work piece relative permeability and electrical conductivity are $\mu_{R}=60, \sigma=4.032 .10^{6 \mathrm{~S}} / \mathrm{m}$ where these properties for ferrite are $\mu_{R}=1000$ and $\sigma=100 \mathrm{~S} / \mathrm{m}$. A thermal insulator layer is considered to prevent the heat exchange between the created joule heating inside the coil and the glass. The alternating current produces alternating magnetic field in the work piece which induces currents due to the Lenz's law.

The induced eddy current on the bottom surface of the work piece is responsible for heat generation. The convective cooling boundary condition with $h=10 \mathrm{~W} / \mathrm{m}^{2} . \mathrm{K}$ is assigned to the top surface of the work piece and glass.

The eigenvalues and eigenfuntions are evaluated numerically in both work piece and glass domains in 3D using Comsol Multiphysics software. In order to be consistent with previous case study the number of evaluated eigenvalues is fixed to 6 . However, the number of boundary 


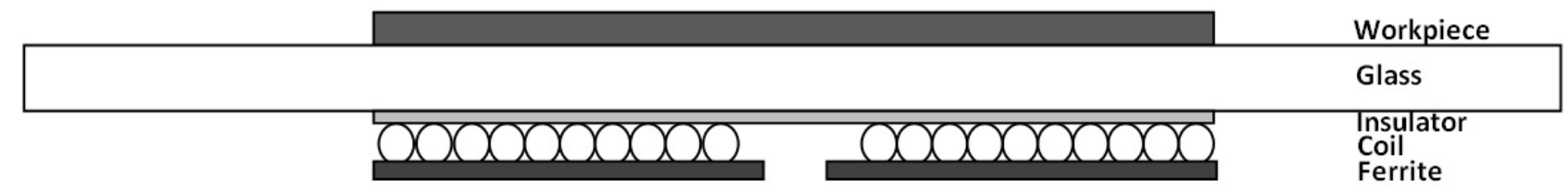

Figure 6. Cook Top geometry cross section diagram

measurement points is varied among 3,5 and 8. Although many different measurement spots can be defined for positioning the sensors, here for the sake of comparison a circle of $50 \mathrm{~mm}$ radius has been chosen and the points are considered on the perimeter of this circle having equal distance from each other. Having the measured data in hand, the temperature is evaluated on the top surface of the work piece located $7 \mathrm{~mm}$ above the measured surface. The 6 computed eigenfunctions along with their corresponding eigenvalues plotted in Fig. 7.

It is obvious from Fig. 7 that the eigenfunction corresponding to the smallest eigenvalue is related to steady state response of the system. An 80 point grid is defined on the top surface of the work piece and the simulation has been done for $600 \mathrm{~s}$.

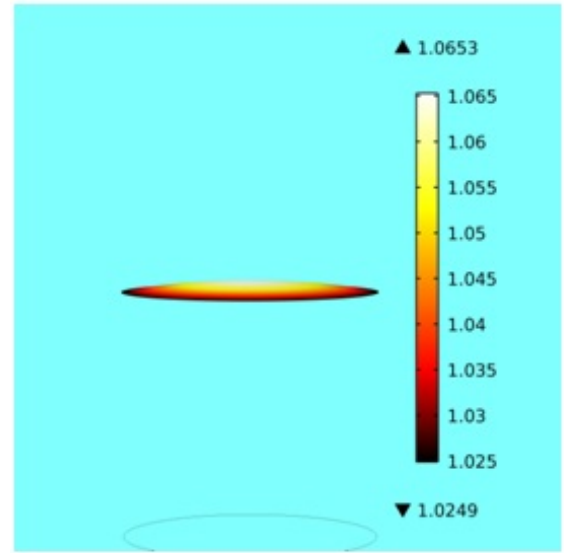

(a)

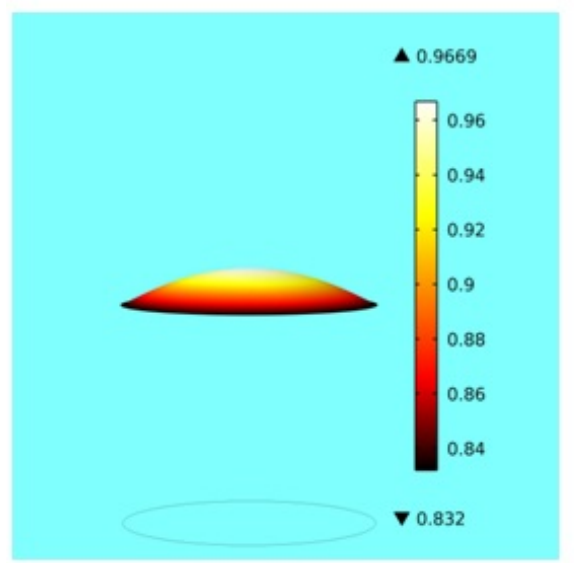

(d)

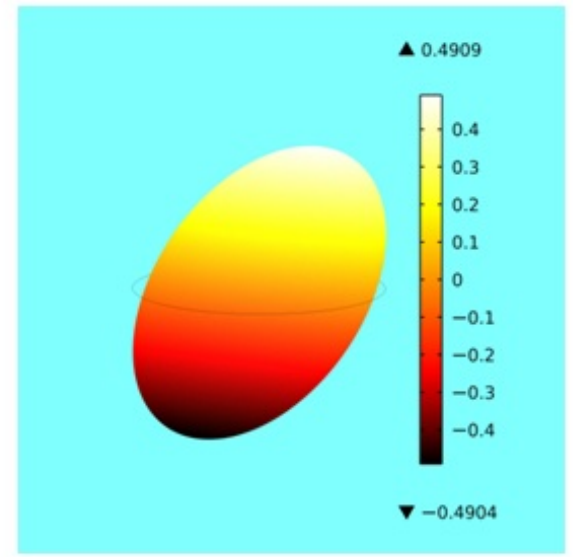

(b)

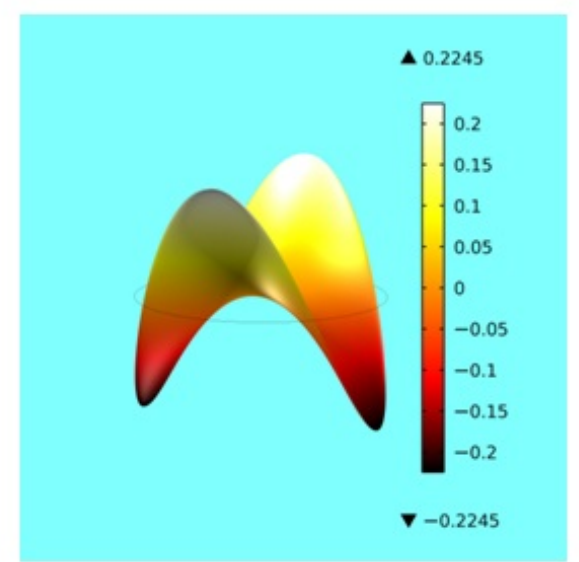

(e)
The infinity norm of error -between the reconstructed temperature among all the grid points and the corresponding actual temperatures- over all grid points of the work piece is computed. Fig. 8 depicts the relative maximum error with respect to the average temperature of all grid points at each $60 \mathrm{~s}$. As it can be observed the relative error for all the three groups of measurement points reduces to almost $4 \%$ at $t=600 \mathrm{~s}$. However, in order to take in to account the noise in data acquisition process and robustness of the method to perturbations a normal distributed random noise is added to the measured data having a mean of $\mu=0{ }^{\circ} \mathrm{C}$ and a standard deviation of $\sigma=2{ }^{\circ} \mathrm{C}$. This time reconstructed temperature is created using the coefficients which are computed from noisy boundary measurements. The procedure has been

Figure 7. The first six eigenfuctions evaluated at top surface of the workpiece. (a) $\lambda_{1}=9 e-4$; (b) $\lambda_{2}=0.001515$ (c) $\lambda_{3}=0.001515$ (d) $\lambda_{4}=0.001577$; (e) $\lambda_{5}=0.001661 ;$ (f) $\lambda_{6}=0.001661$ 


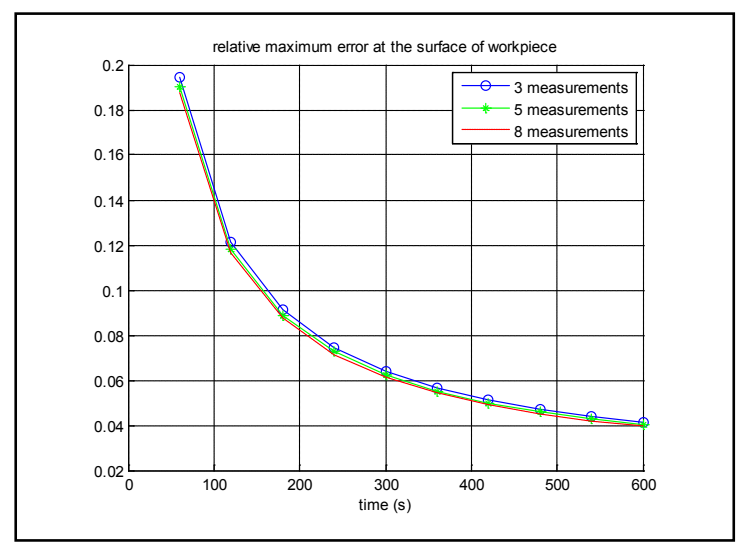

Figure 8. Estimated temperature with 3,5 and 8 measurements

repeated for 100 times for each 60s time interval and the infinity norm of the error between the actual temperature and reconstructed temperature $\|e\|_{\infty}$ is calculated and averaged.

Fig. 9.a indicates the relative averaged maximum error over the 100 repetitions, where Fig. 9.b plots the standard deviation of averaged infinity norm as a function of time. It is clear from Fig. 9 that with increasing the number of measurement data the maximum error in the reconstructed temperature of the surface decreases while the accuracy becomes higher.

\section{CONCLUSIONS AND FUTURE WORK}

This paper tries to develop the description of the basic steps of the method through applying it firstly to a simple example followed by a more complex example in three dimensional space. Based on the results one should keep in mind that although via this method even with few measurement points an acceptable relative error level can be reached but in the presence of corrupting noise extra care should be taken since the fewer the measurement points the higher the standard deviation will be. Hence the more reliable and accurate results are achieved at the expense of higher computation burden due to exploiting higher number of sensors. Therefore one should consider in advance the minimum accuracy of the reconstructed temperature that is desired.

It worth mentioning that while the method seems to be applicable in different configurations, it will be even more interesting to perform further tests in order to optimize the effect of choosing the number and the positioning of the necessary sensors. In addition the sensitivity test regarding to the displacement of the work piece should be evaluated. Trying different boundary condition such as radiation will also be among the future work.

\section{REFERENCES}

[1] N.Milosevic, M. Raynaud, "Analytical solution of transient heat conduction in a two-layer anisotropic cylindrical slab excited superficially by a short laser pulse," International Journal of Heat and Mass Transfer, vol. 47, 2004.

[2] H. Salt, "Transient conduction in two-dimensional composite slabI. Theoretical development of temperature modes," International Journal of Heat and Mass Transfer, vol. 26,1983, pp. 1611-1616.

[3] H. Salt, "Transient conduction in two-dimensional composite slab-II. Physical interpretation of temperature modes," International Journal of Heat and Mass Transfer, vol. 26,1983, pp. 1617-1623.

[4] M.F Abdul Azeez, A.F. Vakakis. "Axisymmetric transient solutions of the heat diffusion problem in layered composite media," International Journal of Heat and Mass Transfeer, vol. 43, 2000, pp. 3883-3895.

[5] Mark S. Gockenbach, Partial Differential Equations, 2nd ed., Society for Industrial and Applied Mathematics (SIAM), PA: Philadelphia, 2010.

[6] M.A.Al-Gwaiz, Strum-Liouville Theory and Its Applications, SpringerVerlag, London, 2008.

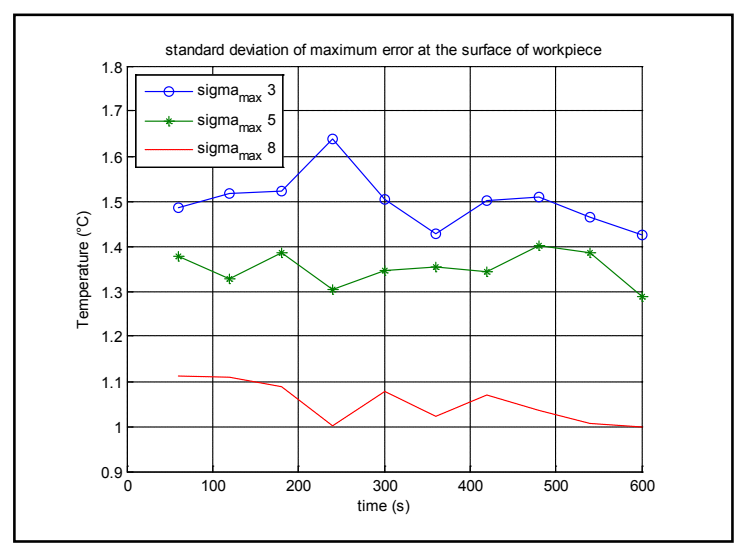

Figure 9. (a) The mean of maximum error of the surface at each $60 \mathrm{~s}$ (b) The standard deviation of maximum error of the surface at each $60 \mathrm{~s}$ 